J. Korean Math. Soc. 42 (2005), No. 5, pp. 913-932

\title{
PSEUDO-SYMMETRIC CONTACT 3-MANIFOLDS
}

\author{
Jong TAEK Cho AND JUN-ICHI INOGUChI \\ Dedicated to Professor Masami Sekizawa on the occasion of his sixtieth birthday
}

\begin{abstract}
Contact Homogeneous 3-manifolds are pseudo-symmetric spaces of constant type. All Sasakian 3-manifolds are pseudo-symmetric spaces of constant type.
\end{abstract}

\section{Introduction}

A Riemannian manifold $(M, g)$ is said to be semi-symmetric if $R \cdot R=$ 0 , where $R$ is the Riemannian curvature tensor and $R \cdot R$ is the derivative of $R$ by $R$ (see section 1 ). Obviously, locally symmetric spaces are semisymmetric.

As a generalization of the semi-symmetry, R. Deszcz[13] introduced the notion of pseudo-symmetry. A Riemannian manifold $(M, g)$ is said to be pseudo-symmetric if there exists a function $L$ such that $R(X, Y) \cdot R=$ $L\{(X \wedge Y) \cdot R\}$ for all vector fields $X$ and $Y$ on $M$. Here $X \wedge Y$ is the endomorphism field defined by

$$
(X \wedge Y) Z=g(Y, Z) X-g(Z, X) Y .
$$

A pseudo-symmetric space $(M, g)$ is said to be proper if $M$ is not semisymmetric. In particular, a pseudo-symmetric space is called a pseudosymmetric space of constant type if $L$ is a constant. Semi-symmetric spaces are pseudo-symmetric spaces of constant type with $L=0$. Threedimensional pseudo-symmetric spaces of constant type have been studied extensively by O. Kowalski and M. Sekizawa[20]-[23]. N. Hashimoto and M. Sekizawa classified 3-dimensional conformally flat pseudo-symmetric spaces of constant type [15].

Received March 3, 2004.

2000 Mathematics Subject Classification: 53B20, 53C25, 53C30.

Key words and phrases: pseudo-symmetric spaces, contact Riemannian 3-manifolds. 
As is well-known, for a Riemannian 3-manifold, its Riemannian curvature is determined by the Ricci curvature. In fact, the Riemannian curvature tensor $R$ is expressed as

$$
\begin{aligned}
R(X, Y) Z= & S(Y, Z) X-S(Z, X) Y \\
& +g(Y, Z) Q X-g(Z, X) Q Y-\frac{\mathrm{s}}{2}(X \wedge Y) Z
\end{aligned}
$$

where $S$ is the Ricci tensor, $Q$ is the corresponding Ricci operator and $\mathrm{s}$ is the scalar curvature. This fundamental fact implies that, in 3-dimensional Riemannian geometry, the constancy of the sectional curvature is equivalent to the Einstein condition, i.e., $\rho_{1}=\rho_{2}=\rho_{3}$ for the eigenvalues $\left\{\rho_{j}\right\}$ of the Ricci tensor. Moreover, the pseudo-symmetry is equivalent to the condition: the eigenvalues $\rho_{1}, \rho_{2}, \rho_{3}$ of the Ricci tensor satisfies $\rho_{1}=\rho_{2}$ (up to numeration) in 3-dimension. Thus the pseudosymmetry is a natural generalization of the constant curvature property in 3-dimension.

It is well known that the maximum dimension of the isometry group is 6 in 3-dimensional Riemannian geometry. The maximum dimension is attained by spaces of constant curvature. There is no Riemannian 3manifold with 5-dimensional isometry group. Riemannian 3-manifolds with 4-dimensional isometry group are homogeneous. Moreover, such spaces are locally isometric to one of the following spaces; the special unitary group $\mathrm{SU}(2)$, the Heisenberg group $\mathbb{H}_{3}$, the special linear group $\mathrm{SL}(2, \mathbf{R})$, product spaces $S^{2} \times \mathbf{R}$ or $H^{2} \times \mathbf{R}$. These three Lie groups appear in the several classification tables, eg., naturally reductive Riemannian homogeneous 3-manifolds [33], 3-dimensional Sasakian space forms [6], 3-dimensional D'Atri spaces [18], or the model geometries in the sense of W. M. Thurston[32]. It is straightforward to check that every Riemannian 3-manifold with 4-dimensional isometry group is a pseudo-symmetric space of constant type. (See Appendix.)

In this article, we concentrate on the pseudo-symmetry of contact Riemannian 3-manifolds. In section 2, we shall show that every Sasakian 3 -manifold is pseudo-symmetric of constant type. Next, in section 3, we shall investigate non-Sasakian contact homogeneous 3-manifolds. Our main result is that all the contact homogeneous 3-manifolds are pseudosymmetric spaces of constant type. Furthermore, we exhibit explicit examples of 3-dimensional proper pseudo-symmetric spaces of constant type. 


\section{Preliminaries}

Let $(M, g)$ be a Riemannian manifold with its Levi-Civita connection $\nabla$. Denote by $R$ the Riemannian curvature tensor of $M$ :

$$
R(X, Y)=\left[\nabla_{X}, \nabla_{Y}\right]-\nabla_{[X, Y]}, \quad X, Y \in \mathfrak{X}(M) .
$$

Here $\mathfrak{X}(M)$ is the Lie algebra of all vector fields on $M$. A tensor field $F$ of type $(1,3)$;

$$
F: \mathfrak{X}(M) \times \mathfrak{X}(M) \times \mathfrak{X}(M) \rightarrow \mathfrak{X}(M)
$$

is said to be curvature-like provided that $F$ has the symmetric properties of $R$. For example,

$$
(X \wedge Y) Z=g(Y, Z) X-g(Z, X) Y, \quad X, Y \in \mathfrak{X}(M)
$$

defines a curvature-like tensor field on $M$. Note that the curvature tensor $R$ of a Riemannian manifold $(M, g)$ of constant curvature $c$ satisfies the formula $R(X, Y)=c(X \wedge Y)$.

As is well known, a curvature-like tensor field $F$ acts on the algebra $\mathcal{T}_{s}^{1}(M)$ of all tensor fields on $M$ of type $(1, s)$ as a derivation ([25], p.44):

$$
\begin{aligned}
& (F \cdot P)\left(X_{1}, \cdots, X_{s} ; Y, X\right) \\
= & F(X, Y)\left\{P\left(X_{1}, \cdots, X_{s}\right)\right\}-\sum_{j=1}^{s} P\left(X_{1}, \cdots, F(X, Y) X_{j}, \cdots, X_{s}\right),
\end{aligned}
$$

where $X_{1}, \cdots, X_{s} \in \mathfrak{X}(M), P \in \mathcal{T}_{s}^{1}(M)$. The derivative $F \cdot P$ of $P$ by $F$ is a tensor field of type $(1, s+2)$.

For a tensor field $P$ of type $(1, s)$, we denote by $\mathcal{Q}(g, P)$ the derivative of $P$ with respect to the curvature-like tensor defined by (1.1);

$$
\begin{aligned}
\mathcal{Q}(g, P)\left(X_{1}, \cdots, X_{s} ; Y, X\right)= & (X \wedge Y) P\left(X_{1}, \cdots, X_{s}\right) \\
& -\sum_{j=1}^{s} P\left(X_{1}, \cdots,(X \wedge Y) X_{j}, \cdots, X_{s}\right) .
\end{aligned}
$$

A Riemannian manifold $(M, g)$ is said to be semi-symmetric if $R \cdot R=0$. Obviously, locally symmetric spaces $(\nabla R=0)$ are semi-symmetric. 
R. Deszcz[13] introduced the notion of a pseudo-symmetric space. A Riemannian manifold $(M, g)$ is said to be pseudo-symmetric if

$$
R \cdot R=L \mathcal{Q}(g, R)
$$

for some function $L$. In particular, if $L$ is constant, $M$ is called a pseudosymmetric space of constant type [21]. A pseudo-symmetric space is said to be proper if it is not semi-symmetric.

For Riemannian 3-manifolds, the following characterizations of the pseudo-symmetry are known (cf. [12, 21, 22]).

Proposition 1.1. A Riemannian 3-manifold $(M, g)$ is pseudo-symmetric if and only if it is quasi-Einstein. Namely, there exists a one-form $\omega$ such that the Ricci tensor field $S$ has the form:

$$
S=a g+b \omega \otimes \omega,
$$

where $a$ and $b$ are functions.

Proposition 1.2. Let $(M, g)$ be a Riemannian 3-manifold. Then $(M, g)$ is a pseudo-symmetric space of constant type if and only if there exists a one-form $\omega$ such that the Ricci tensor $S$ is expressed as $S=$ $a g+b \omega \otimes \omega$, where $a$ is a function and $b$ is a constant.

REMARK 1.3. The preceding proposition can be rephrased as follows (see [21], Proposition 0.1):

A Riemannian 3-manifold is a pseudo-symmetric space of constant type with $R \cdot R=L \mathcal{Q}(g, R)$ if and only if the eigenvalues of the Ricci tensor locally satisfy the following relations (up to numeration):

$$
\left.\rho_{1}=\rho_{2}, \quad \rho_{3}=2 L \text { (constant }\right) .
$$

\section{Contact manifolds}

Let $M$ be a 3-dimensional manifold. A contact form is a one-form $\eta$ such that $\eta \wedge d \eta \neq 0$ on $M$. A 3-manifold $M$ together with a contact form $\eta$ is called a contact 3-manifold. The Reeb vector field $\xi$ is the unique vector field satisfying $\eta(\xi)=1, d \eta(\xi, \cdot)=0$. 
On a contact 3-manifold $(M, \eta)$, there exists a $(1,1)$-tensor field $\varphi$ and a Riemannian metric $g$ such that

$$
\begin{gathered}
\varphi^{2}=-I+\eta \otimes \xi, \quad g(\varphi X, \varphi Y)=g(X, Y)-\eta(X) \eta(Y), \\
g(X, \varphi Y)=d \eta(X, Y), \quad X, Y \in \mathfrak{X}(M) .
\end{gathered}
$$

The structure $(\varphi, \xi, \eta, g)$ is called the associated contact Riemannian structure of $(M, \eta)$. A contact 3-manifold together with its associated contact Riemannian structure is called a contact Riemannian 3manifold. A contact Riemannian 3-manifold $M$ satisfies the following formula [31]:

$$
\left(\nabla_{X} \varphi\right) Y=g(X+\mathrm{h} X, Y) \xi-\eta(Y)(X+\mathrm{h} X), \quad X, Y \in \mathfrak{X}(M) .
$$

Here $\mathrm{h}$ is an endomorphism field defined by $\mathrm{h}=1 / 2 L_{\xi} \varphi$. For later use, we introduce the tensor field $\tau$ of type $(0,2)$ by $\tau=L_{\xi} g$.

The Webster scalar curvature $W$ of a contact Riemannian 3-manifold is defined by

$$
W=\frac{1}{8}(\mathrm{~s}-S(\xi, \xi)+4) .
$$

The torsion invariant of $M$ introduced by S. S. Chern and R. S. Hamilton [9] is the square norm $|\tau|^{2}$ of $\tau$. This invariant can be computed as:

$$
|\tau|^{2}=-2 S(\xi, \xi)+4
$$

A contact Riemannian 3-manifold is said to be an $\eta$-Einstein manifold if the Ricci operator $Q$ has the form:

$$
Q=\alpha I+\beta \eta \otimes \xi
$$

for some functions $\alpha$ and $\beta$.

By the definition, $\eta$-Einstein contact Riemannian 3-manifolds are pseudo-symmetric.

Definition 2.1. [3] A contact Riemannian manifold $M$ is said to be a contact $(\kappa, \mu)$-space if there exist real constants $\kappa$ and $\mu$ such that

$$
\begin{aligned}
R(X, Y) \xi= & \kappa\{\eta(Y) X-\eta(X) Y\} \\
& +\mu\{\eta(Y) \mathrm{h} X-\eta(X) \mathrm{h} Y\}, X, Y \in \mathfrak{X}(M) .
\end{aligned}
$$


A contact 3-manifold $(M, \varphi, \xi, \eta, g)$ is called a Sasakian manifold if it satisfies

$$
\left(\nabla_{X} \varphi\right) Y=g(X, Y) \xi-\eta(Y) X
$$

for all $X, Y \in \mathfrak{X}(M)$.

The formulae (2.3) and (2.6) imply that a contact Riemannian 3manifold is Sasakian if and only if its Reeb vector field $\xi$ is a Killing vector field (cf. [29]).

We easily check that Sasakian manifolds are contact $(\kappa, \mu)$-spaces with $\kappa=1$ and $\mathrm{h}=0$.

Sasakian 3-manifolds have some remarkable properties. For instance, the Ricci operator $Q$ commutes with $\varphi$, i.e., $Q \varphi=\varphi Q$. Moreover $Q$ has the form

$$
Q=\alpha I+\beta \eta \otimes \xi, \quad \alpha=\frac{\mathrm{s}}{2}-1, \beta=3-\frac{\mathrm{s}}{2},
$$

where $s$ is the scalar curvature. Thus the principal Ricci curvatures are

$$
\rho_{1}=\rho_{2}=\frac{\mathrm{s}}{2}-1, \rho_{3}=2 \text {. }
$$

Hence Sasakian 3-manifolds are pseudo-symmetric spaces of constant type.

A plane section $\Pi_{x}$ at a point $x$ of a contact Riemannian 3-manifold is called a holomorphic plane if it is invariant under $\varphi_{x}$. The sectional curvature function of holomorphic planes is called the holomorphic sectional curvature.

A 3-dimensional contact Riemannian manifold with constant holomorphic sectional curvature is called a 3-dimensional contact Riemannian space form [10]. In particular, Sasakian 3-manifolds of constant holomorphic sectional curvature are called 3-dimensional Sasakian space forms.

Three-dimensional contact Riemannian space forms are locally homogeneous. (See [10]).

The first named author obtained the following result.

Proposition 2.2. [10] All 3-dimensional non-Sasakian contact ( $\kappa$, $\mu)$-spaces have constant holomorphic sectional curvature $-(\kappa+\mu)$.

The formula (2.7) implies the following 
Proposition 2.3. A Sasakian 3-manifold is of constant holomorphic sectional curvature if and only if it has constant scalar curvature. In particular, a Sasakian 3-manifold which is a homogeneous Riemannian 3-manifold is of constant holomorphic sectional curvature.

Simply connected and complete 3-dimensional Sasakian space forms are classified as follows:

Proposition 2.4. [6] Simply connected and complete 3-dimensional Sasakian space forms $\mathcal{M}^{3}(c)$ of constant holomorphic sectional curvature $c$ are isomorphic to one of the following unimodular Lie groups with left invariant Sasakian structures: the special unitary group $\mathrm{SU}(2)$ for $c>-3$, the Heisenberg group for $c=-3$, or the universal covering group $\widetilde{\mathrm{SL}}(2, \mathbf{R})$ of the special linear group $\mathrm{SL}(2, \mathbf{R})$ for $c<-3$. The Sasakian space form $\mathcal{M}^{3}(1)$ is the unit 3 -sphere $S^{3}$ with the canonical Sasakian structure.

As a Riemannian 3-manifold, 3-dimensional Sasakian space form is a naturally reductive homogeneous space. The naturally reductive homogeneous representations of the above model spaces (except $S^{3}$ ) are given by

$$
\mathrm{SU}(2) \ltimes \mathrm{U}(1) / \mathrm{U}(1), \quad \mathbb{H}_{3} \ltimes \mathrm{SO}(2) / \mathrm{SO}(2), \quad \widetilde{\mathrm{SL}}(2, \mathbf{R}) \ltimes \mathrm{SO}(2) / \mathrm{SO}(2) .
$$

Next, we recall the following results due to D. E. Blair, Th. Kouforgiorgos, and R. Sharma:

Proposition 2.5. [4] Let $M$ be a contact Riemannian 3-manifold. Then the following three conditions are mutually equivalent:

(i) $M$ is $\eta$-Einstein;

(ii) $Q \varphi=\varphi Q$;

(iii) $M$ is a contact $(\kappa, 0)$-space with $\kappa \leq 1$.

Proposition 2.6. [4] Let $M$ be a contact Riemannian 3-manifold. Then $M$ satisfies $Q \varphi=\varphi Q$ if and only if $M$ is either (i) a Sasakian 3-manifold, (ii) a flat contact Riemannian 3-manifold, or (iii) a nonSasakian contact Riemannian space form of constant holomorphic sectional curvature $-\kappa$ and constant $\xi$-sectional curvature $\kappa$. In the third case, $\kappa<1$.

These propositions imply the following result. 
Corollary 2.7. Contact Riemannian 3-manifolds such that $Q \varphi=$ $\varphi Q$ are pseudo-symmetric. In particular, every Sasakian 3-manifold is a pseudo-symmetric space of constant type.

For explicit Sasakian structure of $\operatorname{SL}(2, \mathbf{R})$, we refer to [17].

REMARK 2.8. A Sasakian manifold (of general dimension) is semisymmetric if and only if is of constant curvature 1 ([28]). Hence every Sasakian 3-manifold, other than space of constant curvature 1, is "proper" pseudo-symmetric space. S. Tanno[30] showed that conformally flat Sasakian 3-manifolds are of constant curvature 1. Thus there are no Sasakian examples in the classification table of conformally flat proper pseudo-symmetric spaces of constant type due to N. Hashimoto and M. Sekizawa[15].

\section{Non-Sasakian contact homogeneous 3-manifolds}

In this section, we study the pseudo-symmetry of contact homogeneous Riemannian 3-manifolds. A contact Riemannian 3-manifold is said to be homogeneous if there exists a connected Lie group $G$ acting transitively as a group of isometries on it which preserve the contact form.

D. Perrone has proven that simply connected contact homogeneous Riemannian 3-manifolds are Lie groups together with left invariant contact Riemannian structures. Moreover such homogeneous spaces are classified by the Webster scalar curvature $W$ and the torsion invariant $|\tau|^{2}$ as follows:

Proposition 3.1. [27] Let $(M, \eta, g)$ be a simply connected contact homogeneous Riemannian 3-manifold. Then $M$ is a Lie group $G$ together with a left invariant contact Riemannian structure $(\eta, g)$.

If $G$ is unimodular, then $G$ is one of the following:

(1) the Heisenberg group $\mathbb{H}_{3}$ if $W=|\tau|=0$;

(2) $\mathrm{SU}(2)$ if $4 \sqrt{2} W>|\tau|$;

(3) $\widetilde{E}(2)$ if $4 \sqrt{2} W=|\tau|>0$;

(4) $\widetilde{\mathrm{SL}}(2, \mathbf{R})$ if $-|\tau| \neq 4 \sqrt{2} W<|\tau|$;

(5) $E(1,1)$ if $4 \sqrt{2} W=-|\tau|<0$.

The Lie algebra $\mathfrak{g}$ of $G$ is generated by an orthonormal basis $\left\{e_{1}, e_{2}=\right.$ $\left.\varphi e_{1}, e_{3}=\xi\right\}$ with commutation relation:

$$
\left[e_{1}, e_{2}\right]=2 e_{3},\left[e_{2}, e_{3}\right]=c_{2} e_{1},\left[e_{3}, e_{1}\right]=c_{3} e_{2} .
$$


If $G$ is non-unimodular, then the Lie algebra $\mathfrak{g}$ of $G$ satisfies the commutation relations:

$$
\left[e_{1}, e_{2}\right]=\alpha e_{2}+2 e_{3},\left[e_{2}, e_{3}\right]=0,\left[e_{3}, e_{1}\right]=\gamma e_{2},
$$

where $e_{3}=\xi, e_{1}, e_{2} \in$ Ker $\eta, e_{2}=\varphi e_{1}, \alpha \neq 0$ and $4 \sqrt{2} W<|\tau|$. If $\gamma=0$, then the structure is Sasakian $(\tau=0)$ and $W=-\alpha^{2} / 4$.

Now we investigate the pseudo-symmetry condition on a unimodular Lie group $G$ with a left invariant non-Sasakian contact Riemannian structure. Then, by Proposition 3.1, there exists an orthonormal basis $\left\{e_{1}, e_{2}=\varphi e_{1}, e_{3}=\xi\right\}$ which satisfies the commutation relations (3.1). By the assumption "non-Sasakian", the case that $c_{2}=c_{3}$ is excluded.

By using the well-known Koszul formula, the connection coefficients $\left\{\Gamma_{i j k}\right\}$ of $(M, g)$ are computed explicitly as follows:

$$
\left\{\begin{array}{l}
\Gamma_{123}=\frac{1}{2}\left(c_{3}-c_{2}+2\right) \\
\Gamma_{213}=\frac{1}{2}\left(c_{3}-c_{2}-2\right) \\
\Gamma_{312}=\frac{1}{2}\left(c_{3}+c_{2}-2\right) \\
\text { all others are zero. }
\end{array}\right.
$$

Here we used the convention: $\Gamma_{i j k}:=g\left(\nabla_{e_{i}} e_{j}, e_{k}\right)$. Then, using (3.2), by straightforward computations we find

$$
\begin{aligned}
& R\left(e_{1}, e_{2}\right) e_{2}=\left(\frac{1}{4}\left(c_{3}-c_{2}\right)^{2}+\left(c_{3}+c_{2}\right)-3\right) e_{1} \\
& R\left(e_{1}, e_{3}\right) e_{3}=\left(-\frac{1}{4}\left(c_{3}-c_{2}\right)^{2}-\frac{1}{2}\left(c_{3}^{2}-c_{2}^{2}\right)+1-c_{2}+c_{3}\right) e_{1} \\
& R\left(e_{2}, e_{1}\right) e_{1}=\left(\frac{1}{4}\left(c_{3}-c_{2}\right)^{2}-3+c_{3}+c_{2}\right) e_{2} \\
& R\left(e_{2}, e_{3}\right) e_{3}=\left(\frac{1}{4}\left(c_{3}+c_{2}\right)^{2}-c_{2}^{2}+1+c_{2}-c_{3}\right) e_{2}, \\
& R\left(e_{3}, e_{1}\right) e_{1}=\left(-\frac{1}{4}\left(c_{3}-c_{2}\right)^{2}-\frac{1}{2}\left(c_{3}^{2}-c_{2}^{2}\right)+1-c_{2}+c_{3}\right) e_{3} \\
& R\left(e_{3}, e_{2}\right) e_{2}=\left(\frac{1}{4}\left(c_{3}+c_{2}\right)^{2}-c_{2}^{2}+1+c_{2}-c_{3}\right) e_{3} .
\end{aligned}
$$


By using (3.3) we get

$$
Q e_{1}=F_{1} e_{1}, Q e_{2}=F_{2} e_{2}, Q e_{3}=F_{3} e_{3},
$$

where we put

$$
\begin{aligned}
& F_{1}=-\frac{1}{2}\left(c_{3}^{2}-c_{2}^{2}\right)-2+2 c_{3} \\
& F_{2}=\frac{1}{2}\left(c_{3}^{2}-c_{2}^{2}\right)-2+2 c_{2} \\
& F_{3}=-\frac{1}{2}\left(c_{3}-c_{2}\right)^{2}+2 .
\end{aligned}
$$

The Webster curvature and torsion invariant are given by

$$
W=\frac{1}{4}\left(c_{2}+c_{3}\right), \quad|\tau|^{2}=\left(c_{2}-c_{3}\right)^{2} .
$$

Let $\left\{\omega^{1}, \omega^{2}, \omega^{3}\right\}$ be the dual orthonormal basis of $\left\{e_{1}, e_{2}, e_{3}\right\}$. Now we suppose that $G$ is pseudo-symmetric, i.e., $Q=a I+b \omega \otimes \zeta$ for some functions $a$ and $b$. If $b=0$, then $G$ is of constant curvature 1 or 0 ([5]). In the former case, $G$ is (locally) isomorphic to $S^{3}$ with canonical Sasakian structure, or equivalently, $\mathrm{SU}(2)$ with biinvariant Sasakian structure.

In the latter case, $G$ is the locally isomorphic to the Euclidean motion group $E(2)$ with flat left invariant contact Riemannian structure. The flat left invariant contact Riemannian structure on $E(2)$ is given explicitly in Example 3.9 below (see also [16], Section 6).

Hereafter, we restrict our attention to the case $b \neq 0$. Then we may have the following three cases:

(1) $Q=a I+b \eta \otimes \xi\left(G\right.$ is $\eta$-Einstein) and $F_{1}=F_{2}=a, F_{3}=a+b$. In this case, we have the commutation relations:

$$
\left[e_{1}, e_{2}\right]=2 e_{3},\left[e_{2}, e_{3}\right]=c_{2} e_{1},\left[e_{3}, e_{1}\right]=\left(2-c_{2}\right) e_{2}
$$

The Ricci operator is given by $Q=\left\{-2\left(1-c_{2}\right)^{2}+2\right\} \eta \otimes \xi$ with $c_{2} \neq 0,2$ (by our assumption $b \neq 0$ ). By the Milnor's result $([24])$ we see that $G$ is locally isometric to $\mathrm{SU}(2)$ (or $\mathrm{SO}(3)$ ) or $\mathrm{SL}(2, \mathbf{R})$ (or $\mathrm{O}(1,2)$ ). In this case, $W=1 / 2$ and $|\tau|^{2}=4\left(c_{2}-1\right)^{2}$.

(2) $Q=a I+b \omega^{2} \otimes e_{2}$ and $F_{1}=F_{3}=a, F_{2}=a+b$. This case has the following two possibilities:

$$
\left[e_{1}, e_{2}\right]=2 e_{3},\left[e_{2}, e_{3}\right]=c_{2} e_{1},\left[e_{3}, e_{1}\right]=\left(c_{2}+2\right) e_{2},
$$


with Ricci operator $Q=4 c_{2} \omega^{2} \otimes e_{2}$ with $c_{2} \neq 0$ (by the assumption $b \neq 0)$.

(ii):

$$
\left[e_{1}, e_{2}\right]=2 e_{3},\left[e_{2}, e_{3}\right]=2 e_{1},\left[e_{3}, e_{1}\right]=c_{3} e_{2},
$$

with Ricci operator $Q=\left(-\frac{1}{2} c_{3}^{2}+2 c_{3}\right) I+\left(c_{3}^{2}-2 c_{3}\right) \omega^{2} \otimes e_{2}$. Since we assumed that $b \neq 0$, we have $c_{3} \neq 0$. Moreover $c_{3} \neq 2$, because we assumed that $G$ is non-Sasakian.

In the former subcase (i), the possible Lie algebras are $\mathfrak{s u}(2), \mathfrak{s l}(2, \mathbf{R})$, or $\mathfrak{e}(1,1)$. In the latter subcase (ii), $\mathfrak{g}$ is isomorphic to $\mathfrak{s u}(2)$ or $\mathfrak{s l}(2, \mathbf{R})$. Hence $G$ is locally isometric to $\mathrm{SU}(2)$ (or $\mathrm{SO}(3)$ ), $\mathrm{SL}(2, \mathbf{R})$ (or $\mathrm{O}(1,2)$ ), or $E(1,1)$.

(3) $Q=a I+b \omega^{1} \otimes e_{1}$ and $F_{2}=F_{3}=a, F_{1}=a+b$; This case has the following two subcases:

(iii): $\quad\left[e_{1}, e_{2}\right]=2 e_{3},\left[e_{2}, e_{3}\right]=c_{2} e_{1},\left[e_{3}, e_{1}\right]=\left(c_{2}-2\right) e_{2}$,

with the Ricci operator $Q=4\left(c_{2}-2\right) \omega^{1} \otimes e_{1}$ with $c_{2} \neq 2$, or (iv):

$$
\left[e_{1}, e_{2}\right]=2 e_{3},\left[e_{2}, e_{3}\right]=c_{2} e_{1},\left[e_{3}, e_{1}\right]=2 e_{2}
$$

with $Q=\left(-\frac{1}{2} c_{2}^{2}+2 c_{2}\right) I+\left(c_{2}^{2}-2 c_{2}\right) \omega^{1} \otimes e_{1}$. Here $c_{2} \neq 0,2$ from the assumption, $b \neq 0$. Hence $G$ is locally isometric to $\mathrm{SU}(2)$ (or $\mathrm{SO}(3)$ ), $\mathrm{SL}(2, \mathbf{R})$ (or $\mathrm{O}(1,2))$ or $E(1,1)$.

Finally, we consider the non-unimodular Lie group $G$ with left invariant (non-Sasakian) contact Riemannian structures. Then by Proposition 3.1 , there exists an orthonormal basis $\left\{e_{1}, e_{2}=\varphi e_{1}, e_{3}=\xi\right\} \in \mathfrak{g}$ such that

$$
\left[e_{1}, e_{2}\right]=\alpha e_{2}+2 e_{3},\left[e_{2}, e_{3}\right]=0,\left[e_{3}, e_{1}\right]=\gamma e_{2},
$$

where $\alpha \neq 0$. Moreover, $G$ is Sasakian if and only if $\gamma=0$. From (3.6), by using the Koszul formula we have

$$
\left\{\begin{array}{l}
\Gamma_{123}=\frac{\gamma+2}{2} \\
\Gamma_{212}=-\alpha \\
\Gamma_{213}=\frac{\gamma-2}{2} \\
\Gamma_{312}=\frac{\gamma-2}{2} \\
\text { all others are zero. }
\end{array}\right.
$$


Then, by the definition of the curvature tensor, we have

$$
\begin{aligned}
& R\left(e_{1}, e_{2}\right) e_{2}=\left(\frac{\gamma^{2}+4 \gamma-12}{4}-\alpha^{2}\right) e_{1} \\
& R\left(e_{1}, e_{3}\right) e_{3}=\left(\frac{-3 \gamma^{2}+4 \gamma+4}{4}\right) e_{1} \\
& R\left(e_{2}, e_{1}\right) e_{1}=\left(\frac{\gamma^{2}+4 \gamma-12}{4}-\alpha^{2}\right) e_{2}+\alpha \gamma e_{3} \\
& R\left(e_{2}, e_{3}\right) e_{3}=\frac{(\gamma-2)^{2}}{4} e_{2} \\
& R\left(e_{3}, e_{1}\right) e_{1}=\alpha \gamma e_{2}+\left(\frac{-3 \gamma^{2}+4 \gamma+4}{4}\right) e_{3} \\
& R\left(e_{3}, e_{2}\right) e_{2}=\frac{(\gamma-2)^{2}}{4} e_{3} .
\end{aligned}
$$

From these, we have the Ricci operator

$$
Q e_{1}=f_{11} e_{1}, Q e_{2}=f_{22} e_{2}+f_{32} e_{3}, Q e_{3}=f_{23} e_{2}+f_{33} e_{3},
$$

where we have put

$$
\begin{aligned}
& f_{11}=\left(-\alpha^{2}-2+2 \gamma-\frac{\gamma^{2}}{2}\right), \\
& f_{22}=\left(-\alpha^{2}-2+\frac{\gamma^{2}}{2}\right), \\
& f_{32}=f_{23}=\alpha \gamma, \quad f_{33}=\left(2-\frac{\gamma^{2}}{2}\right) .
\end{aligned}
$$

Then in a similar way as in the unimodular case, we have

$$
Q=f_{11} I+\left(f_{22}+f_{33}-2 f_{11}\right) \sqrt{2}\left(\omega^{2}+\omega^{3}\right) \otimes \frac{1}{\sqrt{2}}\left(e_{2}+e_{3}\right) .
$$

Hence, we have

THEOREM 3.2. Every 3-dimensional unimodular and non-unimodular Lie group with special left-invariant contact Riemannian structure is a pseudo-symmetric space of constant type.

Together with the classification(Proposition 3.1) of contact homogeneous 3 -dimensional manifolds, we obtain 
Proposition 3.3. Three-dimensional contact homogeneous Riemannian manifolds with special left invariant contact Lie group structure are pseudo-symmetric spaces of constant type.

Also, in view of the classification of contact $(\kappa, \mu)$-space in [3], we get

COROLlary 3.4. Three-dimensional non-Sasakian contact $(\kappa, \mu)$-spaces with special left invariant contact Lie group structure are pseudo-symmetric spaces of constant type.

REMARK 3.5. The Ricci operator $Q$ of a 3-dimensional non-Sasakian contact $(\kappa, \mu)$-space is given by [3]:

$$
Q=-I+\mu \mathrm{h}+(2 \kappa+\mu) \eta \otimes \xi .
$$

EXAMPLE 3.6. (Minkowski motion group) Let $G=E(1,1)$ be the Minkowski motion group:

$$
E(1,1)=\left\{\left(\begin{array}{ccc}
e^{z} & 0 & x \\
0 & e^{-z} & y \\
0 & 0 & 1
\end{array}\right) \mid x, y, z \in \mathbf{R}\right\}
$$

equipped with the following left invariant metric:

$$
g_{\lambda}=e^{-2 z} d x^{2}+e^{2 z} d y^{2}+\lambda^{2} d z^{2},
$$

where $\lambda$ is a positive constant. Then $\left(E(1,1), g_{\lambda}\right)$ is a proper irreducible Riemannian 4-symmetric space. Note that $\left(E(1,1), g_{1}\right)$ is the model space Sol of 3-dimensional solvegeometry [32].

The Ricci tensor of this homogeneous space is given by

$$
\rho_{1}=\rho_{2}=0, \rho_{3}=-2 / \lambda^{2} .
$$

Hence $\left(E(1,1), g_{\lambda}\right)$ is proper pseudo-symmetric space of constant type.

Under the homothetic change of the metric

$$
g=\frac{1}{4}\left(e^{-2 z} d x^{2}+e^{2 z} d y^{2}+\lambda^{2} d z^{2}\right),
$$

we obtain a contact homogeneous 3 -manifold $E(1,1)$ with left invariant contact Riemannian structure determined by the metric $g$ and the contact form $\eta=\frac{1}{2}\left(e^{z} d x+e^{-z} d y\right)$. This contact homogeneous 3-manifold is a non-Sasakian contact space form. In particular, $(E(1,1), g, \eta)$ is a proper pseudo-symmetric space. 
REMARK 3.7. The Lie algebra $\mathfrak{e}(1,1)$ of $E(1,1)$ is given explicitly by

$$
\mathfrak{e}(1,1)=\left\{\left(\begin{array}{ccc}
w & 0 & u \\
0 & -w & v \\
0 & 0 & 1
\end{array}\right) \mid u, v, w \in \mathbf{R}\right\}
$$

Take a basis

$$
F_{1}=\left(\begin{array}{lll}
0 & 0 & 1 \\
0 & 0 & 0 \\
0 & 0 & 0
\end{array}\right), F_{2}=\left(\begin{array}{lll}
0 & 0 & 0 \\
0 & 0 & 1 \\
0 & 0 & 0
\end{array}\right), F_{3}=\left(\begin{array}{ccc}
1 & 0 & 0 \\
0 & -1 & 0 \\
0 & 0 & 0
\end{array}\right)
$$

of $\mathfrak{e}(1,1)$. Then the left translated vector fields of $\left\{F_{1}, F_{2}, F_{3}\right\}$ are given by

$$
f_{1}=e^{z} \frac{\partial}{\partial x}, f_{2}=e^{-z} \frac{\partial}{\partial y}, f_{3}=\frac{\partial}{\partial z} .
$$

The dual coframe field is

$$
\omega^{1}=e^{-z} d x, \omega^{2}=e^{z} d y, \omega^{3}=d z .
$$

Now we take the following left invariant vector fields $u_{1}, u_{2}, u_{3}$ :

$$
u_{1}=\frac{1}{\sqrt{2}}\left(-f_{1}+f_{2}\right), u_{2}=\frac{1}{\sqrt{2}}\left(f_{1}+f_{2}\right), u_{3}=f_{3} .
$$

This left invariant frame field satisfies the commutation relations:

$$
\left[u_{1}, u_{2}\right]=0,\left[u_{2}, u_{3}\right]=u_{1},\left[u_{3}, u_{1}\right]=-u_{2} .
$$

We equip a left invariant Riemannian metric on $E(1,1)$ such that $\left\{e_{1}, e_{2}\right.$, $\left.e_{3}\right\}:=\left\{u_{1} / \lambda_{1}, u_{2} / \lambda_{2}, u_{3} / \lambda_{3}\right\}$ is orthonormal, where $\lambda_{1}, \lambda_{2}, \lambda_{3}$ are positive constants. The resulting Riemannian metric is

$$
g_{\left(\lambda_{1}, \lambda_{2}, \lambda_{3}\right)}=\frac{\lambda_{1}^{2}}{2}\left(-\omega^{1}+\omega^{2}\right)^{2}+\frac{\lambda_{2}^{2}}{2}\left(\omega^{1}+\omega^{2}\right)^{2}+\lambda_{3}^{2}\left(\omega^{3}\right)^{2} .
$$

Any left invariant metric on $E(1,1)$ is isometric with one of the following metric:

$$
g_{\left(\lambda_{1}, \lambda_{2}, \lambda_{1} \lambda_{2}\right)}=\frac{\lambda_{1}^{2}}{2}\left(-\omega^{1}+\omega^{2}\right)^{2}+\frac{\lambda_{2}^{2}}{2}\left(\omega^{1}+\omega^{2}\right)^{2}+\frac{1}{\lambda_{1}^{2} \lambda_{2}^{2}}\left(\omega^{3}\right)^{2},
$$


with $\lambda_{1} \geq \lambda_{2}>0$ (see [26], Proposition 2.3). Note that $g_{(1,1, \lambda)}=g_{1}=$ $4 g$.

The commutation relations of $\left\{e_{1}, e_{2}, e_{3}\right\}$ are

$$
\left[e_{1}, e_{2}\right]=c_{3} e_{3},\left[e_{2}, e_{3}\right]=c_{1} e_{1},\left[e_{3}, e_{1}\right]=c_{2} e_{2}
$$

with $c_{1}=1 /\left(\lambda_{2} \lambda_{3}\right), c_{2}=-1 /\left(\lambda_{3} \lambda_{1}\right), c_{3}=0$. It follows also that

$$
\mu_{1}=-\frac{\lambda_{1}+\lambda_{2}}{2 \lambda_{1} \lambda_{2} \lambda_{3}}, \mu_{2}=\frac{\lambda_{1}+\lambda_{2}}{2 \lambda_{1} \lambda_{2} \lambda_{3}}, \mu_{3}=\frac{\lambda_{1}-\lambda_{2}}{2 \lambda_{1} \lambda_{2} \lambda_{3}}
$$

where we have put $\mu_{i}=1 / 2\left(c_{1}+c_{2}+c_{3}\right)-c_{i}$ for $i=1,2,3$. Then we have $\rho_{1}=2 \mu_{2} \mu_{3}, \rho_{2}=2 \mu_{3} \mu_{1}, \rho_{3}=2 \mu_{1} \mu_{2}$ (cf. [24]). From these, we see that $\rho_{1}=\rho_{2}$ if and only if $\lambda_{1}=\lambda_{2}$. The other two cases $\rho_{2}=\rho_{3}$ or $\rho_{3}=\rho_{1}$ can not occur. Hence, we obtain the following result.

Corollary 3.8. Any pseudo-symmetric left invariant Riemannian metrics on $E(1,1)$ is homothetic to the 4-symmetric metric $e^{-2 z} d x^{2}+$ $e^{2 z} d y^{2}+\lambda^{2} d z^{2}$ for some $\lambda>0$.

EXAMPLE 3.9. (Euclidean motion group) The Euclidean motion group $G=E(2)$ is given explicitly by the following matrix group:

$$
E(2)=\left\{\left(\begin{array}{ccc}
\cos \theta & -\sin \theta & x \\
\sin \theta & \cos \theta & y \\
0 & 0 & 1
\end{array}\right) \mid x, y, \theta \in \mathbf{R}\right\} .
$$

Let $\widetilde{G}$ the universal covering group of $E(2)$. Then $\widetilde{G}$ is $\mathbf{R}^{3}(x, y, z)$ with multiplication:

$(x, y, z) \cdot\left(x^{\prime}, y^{\prime}, z^{\prime}\right)=\left(x+\cos z x^{\prime}-\sin z y^{\prime}, y+\sin z x^{\prime}+\cos z y^{\prime}, z+z^{\prime}\right)$.

Take positive constants $\alpha, \beta$ and $\gamma$ and a left invariant frame:

$$
\begin{aligned}
& e_{1}=\beta\left(-\sin z \frac{\partial}{\partial x}+\cos z \frac{\partial}{\partial y}\right), \\
& e_{2}=\gamma \frac{\partial}{\partial z}, \\
& e_{3}=\alpha\left(\cos z \frac{\partial}{\partial x}+\sin z \frac{\partial}{\partial y}\right)=\xi .
\end{aligned}
$$


Then this frame satisfies the following commutation relations:

$$
\left[e_{1}, e_{2}\right]=c_{1} e_{3}, \quad\left[e_{2}, e_{3}\right]=c_{2} e_{1}, \quad\left[e_{3}, e_{1}\right]=0
$$

with $c_{1}=\beta \gamma / \alpha, c_{2}=\alpha \gamma / \beta$. The left invariant Riemannian metric determined by the condition $\left\{e_{1}, e_{2}, e_{3}\right\}$ is orthonormal is given by (cf. $[26])$ :

$$
\begin{aligned}
g_{\alpha, \beta, \gamma}= & \left(\alpha^{-2} \cos ^{2} z+\beta^{-2} \sin ^{2} z\right) d x^{2}+\left(\alpha^{-2}-\beta^{-2}\right) \sin (2 z) d x d y \\
& +\left(\alpha^{-2} \sin ^{2} z+\beta^{-2} \cos ^{2} z\right) d y^{2}+\gamma^{-2} d z^{2}
\end{aligned}
$$

This family essentially exhausts all left invariant metrics on $\widetilde{G}$. See [26], Proposition 2.4.

The principal Ricci curvatures are given by

$\rho_{1}=-\frac{1}{2}\left(c_{1}+c_{2}\right)\left(c_{1}-c_{2}\right), \quad \rho_{2}=-\frac{1}{2}\left(c_{1}-c_{2}\right)^{2}, \quad \rho_{3}=\frac{1}{2}\left(c_{1}+c_{2}\right)\left(c_{1}-c_{2}\right)$.

Hence $\widetilde{G}$ is pseudo-symmetric if and only if $c_{1}=c_{2}$. This condition is equivalent to $\alpha=\beta$, i.e., $\widetilde{G}$ is flat.

We may normalize $\{\alpha, \beta, \gamma\}$ so that $c_{1}=2$ (equivalently, $\alpha=\beta \gamma / 2$ ). Under this normalization, $c:=c_{2}=\gamma^{2} / 2$.

The associated contact Riemannian structure $(\eta, \xi, \varphi)$ is given by

$$
\begin{gathered}
\eta=\alpha^{-1}(\cos z d x+\sin z d y), \quad \xi=e_{1}, \\
\varphi e_{1}=0, \quad \varphi e_{2}=e_{3}, \quad \varphi e_{3}=-e_{2}
\end{gathered}
$$

COROLlary 3.10. Among all the left invariant Riemannian metrics on the universal covering $\widetilde{E}(2)$ of the Euclidean motion group, the flat metrics are only left invariant metrics which are pseudo-symmetric. Hence there are no proper pseudo-symmetric left invariant metric on $\widetilde{E}(2)$.

There exist many homogeneous Riemannian 3-manifolds which are pseudo-symmetric. For instance, in this paper, we exhibit examples of contact homogeneous Riemannian 3-manifolds which are proper pseudosymmetric spaces (of constant type).

On the other hand, O. Kowalski[19] gave examples of non-homogeneous pseudo-symmetric 3-spaces. Non-homogeneous Sasakian 3-manifolds provide examples of non-homogeneous pseudo-symmetric spaces. 
In view of the results of this paper, one may raise the following question :

"Are there examples of non-homogeneous, non-Sasakian, pseudo-symmetric contact manifolds?"

The classification due to O. Kowalski and M. Sekizawa[22, 23] motivates us to study the pseudo-symmetry of confoliated 3-manifolds (in the sense of Y. Eliashberg and W. Thurston[14]).

\section{Appendix. Riemannian 3-manifolds with 4-dimensional isometry group}

As we mentioned in Introduction, every Riemannian 3-manifold with 4-dimensional isometry group is a pseudo-symmetric space.

In this Appendix, we give a proof of this well known fundamental result for reader's convenience.

It is classically known that Riemannian 3-manifolds with 4-dimensional isometry group are homogeneous (E. Cartan[8]).

L. Bianchi[1] and E. Cartan[8] obtained the following two parameter family of homogeneous Riemannian metrics:

$$
g_{\lambda, \mu}=\frac{d x^{2}+d y^{2}}{\left\{1+\mu\left(x^{2}+y^{2}\right)\right\}^{2}}+\left(d z+\frac{\lambda}{2} \frac{y d x-x d y}{1+\mu\left(x^{2}+y^{2}\right)}\right)^{2} .
$$

The metric $g_{\lambda, \mu}$ is defined on the region:

$$
\mathcal{D}=\left\{(x, y, z) \in \mathbf{R}^{3}(x, y, z) \mid 1+\mu\left(x^{2}+y^{2}\right)>0\right\} .
$$

Note that $\mathcal{D}$ is the whole $\mathbf{R}^{3}(x, y, z)$ for $\mu \geq 0$.

The Riemannian 3-manifold $\left(\mathcal{D}, g_{\lambda, \mu}\right)$ is locally isometric to:

(1) $\lambda=\mu=0$ : Euclidean 3-space,

(2) $\lambda=0$ : Riemannian products $S^{2} \times \mathbf{R}(\mu>0)$, or $H^{2} \times \mathbf{R}(\mu<0)$,

(3) $\lambda \neq 0, \mu=0$ : Heisenberg group $\mathbb{H}_{3}$,

(4) $\lambda \neq 0, \mu>0: \mathrm{SU}(2)$,

(5) $\lambda \neq 0, \mu<0: \mathrm{SL}(2, \mathbf{R})$

In particular, $\mathcal{D}$ is of constant positive curvature $\lambda^{2} / 4$ if $4 \mu=\lambda^{2}$ and $\lambda \neq 0$.

Moreover, every Riemannian 3-manifold with 4-dimensional isometry group is locally isometric to $\mathcal{D}$ for some $\lambda, \mu$. 
Take an orthonormal frame field

$$
\begin{aligned}
& e_{1}=\left\{1+\mu\left(x^{2}+y^{2}\right)\right\} \frac{\partial}{\partial x}-\frac{\lambda y}{2} \frac{\partial}{\partial z} \\
& e_{2}=\left\{1+\mu\left(x^{2}+y^{2}\right)\right\} \frac{\partial}{\partial y}+\frac{\lambda x}{2} \frac{\partial}{\partial z} \\
& e_{3}=\frac{\partial}{\partial z}
\end{aligned}
$$

Then the Ricci tensor of $g_{\lambda, \mu}$ is given by $R_{11}=R_{22}=4 \mu-\lambda^{2}, R_{33}=$ $\lambda^{2} / 2$.

Proposition A.1. Every Riemannian 3-manifold with 4-dimensional isometry group is a pseudo-symmetric space of constant type.

Direct computation shows that $R \cdot R=0$ if and only if $\lambda^{2}\left(4 \mu-\lambda^{2}\right)=0$. This relation implies that $\mathcal{D}$ is semi-symmetric if and only if $\mathcal{D}$ is locally symmetric.

Corollary A.2. A Riemannian 3-manifold with 4-dimensional isometry group is semi-symmetric if and only if it is locally symmetric and hence locally isometric to $S^{2} \times \mathbf{R}$ or $H^{2} \times \mathbf{R}$.

Comparing the metrics as above and the classification of 3-dimensional D'Atri space due to O. Kowalski[18], we obtain

Proposition A.3. Three-dimensional D'Atri spaces are pseudo-symmetric spaces of constant type.

REMARK A.4. The dual one-form $\omega^{3}$ of $e_{3}$ is $\omega^{3}=d z+(\lambda / 2)(y d x-$ $x d y) /\left\{1+\mu\left(x^{2}+y^{2}\right)\right\}$. This one-form is contact if and only if $\lambda \neq 0$. Now we assume that $\lambda \neq 0$. Take a contact form $\eta=\lambda \omega^{3} / 2$. Then the associated Riemannian metric is $\hat{g}=\frac{\lambda^{2}}{4} g_{\lambda, \mu}$. The resulting contact Riemannian 3-manifold $(\mathcal{D}, \eta, \hat{g})$ is a Sasakian space form of constant holomorphic sectional curvature $-3+16 \mu / \lambda^{2}$. (Compare with Proposition 3.2.)

Isometry groups of Riemannian 3-manifolds have dimension at most 6. A Riemannian 3-manifold has 6-dimensional isometry group if and only if it is of constant curvature. Moreover, there is no Riemannain 3-manifold with 5-dimensional isometry group.

Corollary A.5. Riemannian 3-manifolds whose isometry groups have dimension $\geq 4$ are pseudo-symmetric spaces of constant type. 


\section{References}

[1] L. Bianchi, Lezioni di Geometrie Differenziale, E. Spoerri Librao-Editore, 1894.

[2] D. E. Blair, Contact Manifolds in Riemannian Geometry, Lecture Notes in Math. 509 (1976), Springer-Verlag, Berlin-Heidelberg-New-York.

[3] D. E. Blair, Th. Koufogiorgos and B. J. Papantoniou, Contact metric manifolds satisfying a nullity condition, Israel J. Math. 91 (1995), 189-214.

[4] D. E. Blair, Th. Koufogiorgos, and R. Sharma, A classification of 3-dimensional contact metric manifolds with $Q \varphi=\varphi Q$, Kōdai Math. J. 13 (1990), 391-401.

[5] D. E. Blair and R. Sharma, Three-dimensional locally symmetric contact metric manifolds, Boll. Unione. Mat. Ital. Sez. A Mat. Soc. Cult. (8) 4 (1990), no. 3, $385-390$.

[6] D. E. Blair and L. Vanhecke, Symmetries and $\varphi$-symmetric spaces, Tôhoku Math. J. 39 (1987), no. 2, 373-383.

[7] G. Calvaruso, D. Perrone, and L. Vanhecke, Homogeneity on three-dimensional contact metric manifolds, Israel J. Math. 114 (1999), 301-321.

[8] E. Cartan, Leçon sur la geometrie des espaces de Riemann, Second Edition, Gauthier-Villards, Paris, 1946.

[9] S. S. Chern and R. S. Hamilton, On Riemannian metrics adapted to threedimensional contact manifolds, Lecture Notes in Math. 1111 (1985), Springer Verlag, 279-305.

[10] J. T. Cho, A class of contact Riemannian manifolds whose associated CRstructure are integrable, Publ. Math. Debrecen 63 (2003), 193-211.

[11] J. T. Cho and L. Vanhecke, Classification of symmetric-like contact metric $(k, \mu)$-spaces, Publ. Math. Debrecen 62 (2003), 337-349.

[12] J. Deprez, R. Deszcz, and L. Verstraelen, Examples of pseudo-symmetric conformally flat warped products, Chinese J. Math. 17 (1989), 51-65.

[13] R. Deszcz, On pseudosymmetric spaces, Bull. Soc. Math. Belg. 44 (1992), 1-34.

[14] Y. Eliashberg and W. Thurston, Confoliations, AMS University Lecture Series 13 (1998).

[15] N. Hashimoto and M. Sekizawa, Three-dimensional conformally flat pseudosymmetric spaces of constant type, Arch. Math. (Brno) 36 (2000), 279-286.

[16] J. Inoguchi, Minimal surfaces in 3-dimensional solvable Lie groups, Chinese Ann. Math. Ser. B 24 (2003), 73-84.

[17] J. Inoguchi, T. Kumamoto, N. Ohsugi, and Y. Suyama, Differential geometry of curves and surfaces in 3-dimensional homogeneous spaces III, Fukuoka Univ. Sci. Rep. 30 (2000), 131-160.

[18] O. Kowalski, Spaces with volume preserving symmetries and related classes of Riemannian manifolds, Rend. Sem. Mat. Univ. Politec. Torino 64 (1983), 131159.

[19] Ricci curvatures $\rho_{1}=\rho_{2} \neq \rho_{3}$, Nagoya Math. J. 132 (1993), 1-36.

[20] O. Kowalski and M. Sekizawa, Local isometry classes of Riemannian 3-manifolds with constant Ricci eigenvalues $\rho_{1}=\rho_{2} \neq \rho_{3}$, Arch. Math. (Brno) 32 (1996), $137-145$.

[21] __. Three-dimensional Riemannian manifolds of c-conullity two, Riemannian Manifolds of Conullity Two, Chapter 11, World Scientific, Singapore, 1996. 
[22] _ Pseudo-symmetric spaces of constant type in dimension three-elliptic spaces, Rend. Mat. Appl. (7) 17 (1997), 477-512.

[23] _ Pseudo-symmetric spaces of constant type in dimension three-nonelliptic spaces, Bull. Tokyo Gakugei Univ. (4) 50 (1998), 1-28.

[24] J. Milnor, Curvature of left invariant metrics on Lie groups, Adv. Math. 21 (1976), 293-329.

[25] B. O'Neill, Semi-Riemannian Geometry with Application to Relativity, Academic Press, Orland, 1983.

[26] V. Patrangenaru, Classifying 3- and 4-dimensional homogeneous Riemannian manifolds by Cartan triples, Pacific J. Math. 173 (1996), 511-532.

[27] D. Perrone, Homogeneous contact Riemannian three-manifolds, Illinois J. Math. 13 (1997), 243-256.

[28] T. Takahashi, Sasakian manifolds with pseudo-Riemannian metric, Tôhoku Math. J. 21 (1969), 271-290.

[29] S. Tanno, Sur une variété de K-contact métrique de dimension 3, C. R. Math. Acad. Sci. Paris 263 (1966), A 317-A319.

[30] L Locally symmetric $K$-contact Riemannian manifolds, Proc. Japan Acad. Ser. A Math. Sci. 43 (1967), 581-583.

[31] _ Variational problems on contact Riemannian manifolds, Trans. Amer. Math. Soc. 314 (1989), 349-379.

[32] W. M. Thurston, Three-dimensional Geometry and Topology I, Princeton Math. Ser., vol. 35 (S. Levy, eds.), 1997.

[33] F. Tricerri and L. Vanhecke, Homogeneous Structures on Riemannian Manifolds, London Math. Soc. Lecture Note Ser. vol. 83, Cambridge Univ. Press, London, 1983.

Jong Taek Cho

Department of Mathematics

Chonnam National University

CNU The Institute of Basic Science

Kwangju 500-757, Korea

E-mail: jtcho@chonnam.ac.kr

Jun-ichi Inoguchi

Department of Mathematics Education

Faculty of Education

Utsunomiya University

Mine 350, Utsunomiya 321-8505, Japan

E-mail: inoguchi@cc.utsunomiya-u.ac.jp 